\title{
Design and fabrication of 3D printed QuadDrone for altitude measurement
}

\section{B. Meenakshipriya, N. Boopathi Raja*, S. Faroshkhan and S. Matris}

\author{
Mechatronics Department, \\ Kongu Engineering College, \\ Perundurai, India \\ Email: b.meenakshipriya@gmail.com \\ Email: boopathikangayam@gmail.com \\ Email: faroshsulthan1299@gmail.com \\ Email: matrisaros55@gmail.com \\ *Corresponding author
}

\begin{abstract}
The main motivation towards the application of the proposed QuadDrone is precise altitude measurement. The QuadDrone is designed using SolidWorks and fabricated using 3D printing technology. The drone body is fabricated using poly lactic acid (PLA) material (black) and acrylonitrile butadiene styrene (ABS) material (white) is used to fabricate drone landing box. For this purpose, Ultimaker and CubePro 3D printers are used. The printed parts are tested for dimensional accuracy and surface roughness. The electronic components used for drone are flight control (KK2), electronic speed controllers (ESCs), transmitter and receiver (six channels), BLDC motors $(2,280 \mathrm{KV})$, propellers $(5045)$ and pressure sensor for altitude measurement (BMP180). The QuadDrone is assembled with 3D printed parts and electronics components. The difficulties encountered while developing the drone are assembly errors, sizing issues and weight balancing. The recent technologies like 3D printing, rapid prototyping and drone technology are used to develop the QuadDrone. The performance to measure the altitude of QuadDrone is tested in the open environment and it will act as a precision altimeter.
\end{abstract}

Keywords: unmanned aerial vehicle; UAV; BMP180; 3D printed drone; drone altitude; PLA-based drone; aerial vehicle.

Reference to this paper should be made as follows: Meenakshipriya, B., Raja, N.B., Faroshkhan, S. and Matris, S. (2021) 'Design and fabrication of 3D printed QuadDrone for altitude measurement', Int. J. Aerospace System Science and Engineering, Vol. 1, No. 1, pp.85-94.

Biographical notes: B. Meenakshipriya completed her PhD in Process Control from Anna University, Chennai (India) in 2012. Her active research areas include nonlinear control, process control, robotics and control. She has published more than 90 articles in various journals and conference proceedings.

N. Boopathi Raja obtained his UG degree in Mechatronics Engineering. His areas of research interest are robotics and automation.

S. Faroshkhan obtained his UG degree in Mechatronics Engineering. His areas of research interest are robotics and automation.

S. Matris obtained his UG degree in Mechatronics Engineering. His areas of research interest are robotics and automation. 


\section{Introduction}

In modern times, the airspace above our heads is not the sole preserve of manned aviation, and it is common to see unmanned aerial vehicles (UAVs), usually referred to as drones or multicopters in airborne operations. UAVs have received much interest from research and business to implement it in various fields of professional, research and hobby activities. The unique selling point of UAVs is that these devices are unmanned. For example (Ganesh et al., 2015), they can operate in disaster areas without harming humans and deploy much faster than human helicopters. In addition, the UAV is much smaller than manned aircraft and can be used in confined areas such as buildings or forests. In recent years, additive manufacturing has also been developing fast-growing technologies. 3D printers are changing the digital design to meet three-dimensional solid objects through the additive process. The aggregation process is a layer-by-layer melting of layers by means of feedstock to form three-dimensional solids. 3D printable models are created with the help of computer aided design (CAD) software or 3D scanning tools. Typically, the height of the drone between the drone and the ground surface is measured with the help of a LIDAR sensor or ultrasonic range finder sensor. The ultrasonic range finder is not suitable for long range, but instead LIDAR can be used because it is a laser-based sensor to measure long distances as shown in Figure 1. The accuracy of measurement using LIDAR is precious, but the cost is high. To overcome this problem, a BMP180 pressure sensor has been added to enable this QuadDrone made by a 3D printer and to measure the height of a drone from some data line.

Figure 1 Height measurement using drone with LIDAR (see online version for colours)

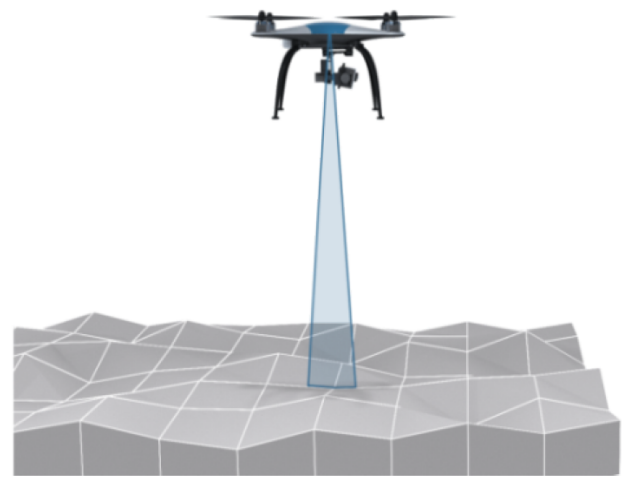

\section{Existing and proposed mobile robot for QuadDrone}

\subsection{Existing height measuring drone}

In this work, we develop crop height measurement using the BMP180 sensor in the QuadDrone and continuously communicate the data measured on the mobile via the Bluetooth module. Getting accurate and timely crops height estimates are important to explain plant health and growth. Nowadays, this data can be measured using manual measurements or by running heavy equipment through the field or using LIDAR-based 
scanners implemented in UAVs to measure height. These collection methods are time consuming and can harm crops and are not used regularly.

The height of the micro-UAV measurement based on the UAV-mounted measurement system (Anthony et al., 2014), which uses a laser scanner to calculate crop height, is shown in Figure 2. The height of the drone in this system is equal to height of the measured plant. Drone height is measured with a laser scanner system. Micro-UAV is flying over the crop to measure crop height. The measurements from the laser scanner must be converted to height readings with the help of filters to filter the output signal and the precise crop height and UV height. In this system, he introduced a high-level algorithm for this process.

Figure 2 Micro-UAV measuring crop heights (see online version for colours)

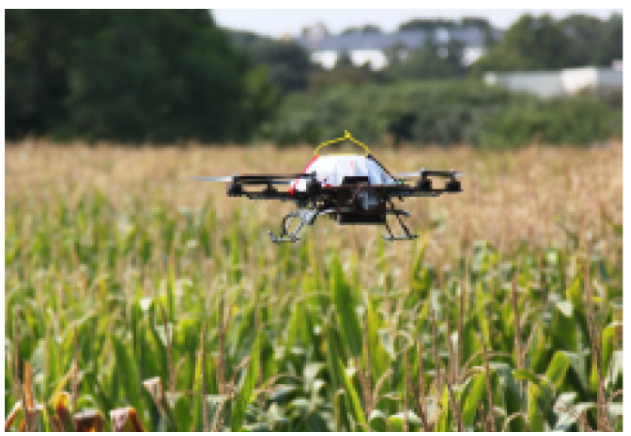

The present work differs from our approach in that we are using BMP180 pressure sensors instead of LIDAR due to the high cost of LIDAR, and the process of collecting the sensor data is much more tedious. We also must fly much closer to the plant to sense the altitude between the ground and the object. QuadDrone move closer to plant for obtain high precise altitude.

\subsection{Proposed QuadDrone}

- 3D model of QuadDrone frame is designed using SolidWorks and fabricated using 3D printer. For this purpose, CubePro and Ultimaker are used. Acrylonitrile butadiene styrene (ABS) and poly lactic acid (PLA) materials are used to develop frame.

- KK2 flight controller (Kale et al., 2015) is used as controller which provides PWM signal to four BLDC motors through electronic speed controllers (ESC) separately. It have inbuilt gyroscope and accelerometer which ensure the stability of QuadDrone.

- $\quad$ BMP180 is employed to measure altitude using drone. Measured altitude transmits to mobile phone through Bluetooth module with delay of five seconds.

- Merits: controller provides good stability, fast and efficient reading provided by BMP180 and altitude reading is also used in safe landing of drone. 


\section{Component specifications}

The model consists of mechanical as well as electrical components such as 3D printed drone frame, BLDC motor, propeller, LiPo batter, ESC, KK2 flight controller, transmitter and receiver $2.4 \mathrm{GHz}$, LiPo connectors, BMP180 sensor, Arduino Nano and Bluetooth module. Table 1 provides the details of components mainly used in the system.

- $\quad$ Mass of the components:

$$
\begin{array}{ll}
\mathrm{a} & \text { material used }=\mathrm{PLA}, \mathrm{ABS} \\
\mathrm{b} & \text { density }=\mathrm{PLA}-1.25 \mathrm{~g} / \mathrm{cm}^{3}, \mathrm{ABS}-1.04 \mathrm{~g} / \mathrm{cm}^{3} \\
\mathrm{c} & \text { frame }=98 \mathrm{~g} \\
\mathrm{~d} & \text { motor mass }=80 \mathrm{~g} \\
\mathrm{e} & \mathrm{ESC}=92 \mathrm{~g} \\
\mathrm{f} & \text { propeller }=80 \mathrm{~g} \\
\mathrm{~g} & \mathrm{KK} 2 \text { controller }=80 \mathrm{~g} \\
\mathrm{~h} & \mathrm{BMP} 180=7 \mathrm{~g} \\
\mathrm{i} & \text { Arduino Nano }=10 \mathrm{~g} \\
\mathrm{j} & \text { Bluetooth }=20 \mathrm{~g} \\
\mathrm{k} & \text { LiPo battery }=200 \mathrm{~g} \\
\mathrm{l} & \text { total mass }(\mathrm{W})=667 \mathrm{~g} \sim 700 \mathrm{~g} .
\end{array}
$$

- Motor specification:

$$
\begin{aligned}
& \text { a motor type: brushless DC motor } \\
& \text { b KV: 2,280 RP-M/V } \\
& \text { c max. watts: } 92 \mathrm{~W} \\
& \text { d max. current: } 8.3 \mathrm{Ah} \\
& \text { e LiPo cells: } 1-4 \mathrm{~S} \\
& \text { f max. thrust: } 400 \mathrm{~g} .
\end{aligned}
$$

- Arduino Nano specification

$$
\begin{array}{ll}
\text { a manufacturer: ATMEL } \\
\text { b model no.: ATMEGA328 } \\
\text { c digital I/O pins: } 14 \text { (6 PWM output) } \\
\text { d } \\
\text { e malogue input pin: } 6 \\
\text { f } \\
\text { g } \\
\text { RAM: } 2 \mathrm{~K} \text { bytes } \\
\text { EEPROM: } 2 \mathrm{~K} \text { bytes. }
\end{array}
$$

- ESC specification:
a input: 30 A continuous (35 Amps for 10 seconds)
b input voltage: $2-4$ cells lithium polymer
c BEC: $5 \mathrm{~V}, 2$ Amp. 
- Transmitter and receiver specification:

a channels: 6

b RF range: $2.40-2.48 \mathrm{GHz}$

c RF power: less than $20 \mathrm{dBm}$

d bandwidth: $500 \mathrm{KHz}$

e band: 160

f operating voltage: $12 \mathrm{~V}$.

Table 1 List of components

\begin{tabular}{|c|c|c|}
\hline S. no & Components & Description \\
\hline 1 & BLDC motor & 11.1 V 25,308 RPM (3S 2,280 KV) \\
\hline 2 & Electronic speed controller (ESC) & $30 \mathrm{Ah}$ \\
\hline 3 & LiPo battery & 11.1 V-2,200 MAh $25 \mathrm{C}$ \\
\hline 4 & Propeller(5045) & 5 inch dia., 4.5 inch pitch \\
\hline 5 & Arduino Nano & Atmega 328P \\
\hline 6 & BMP180 pressure sensor & $\begin{array}{c}300-1,100 \mathrm{hPa}(9,000 \mathrm{~m} \text { to }-500 \mathrm{~m} \text { above sea } \\
\text { level })\end{array}$ \\
\hline 7 & Transmitter and receiver & 2.4 GHz 6-channel PWM signal \\
\hline 8 & Bluetooth module & Up to $10 \mathrm{~m}$ \\
\hline 9 & KK2 flight controller & Up to 8 motors, inbuilt IMU \\
\hline 10 & $3 \mathrm{D}$ printed frame & As per design \\
\hline 11 & LiPo connecter & T type connector \\
\hline 12 & Bolt and nuts & Stainless steel \\
\hline
\end{tabular}

\subsection{Design calculation}

- $\quad$ thrust

Total thrust $=$ No. of motor $\times$ single motor thrust $(\mathrm{g})$

$$
=4 \times 400=1600 \mathrm{~g}
$$

- $\quad$ thrust to weight ratio

$$
\begin{aligned}
\text { Ratio } & =\text { Total thrust } / \text { Weight of drone } \\
& =1600 / 700=2.29
\end{aligned}
$$

- $\quad$ ESC rating

$$
\begin{aligned}
\text { Current } & =1.5 \times \text { Max. current of motor } \\
& =1.5 \times 8.3 \\
& =12.45 \mathrm{~A} \sim 30 \mathrm{Ah}
\end{aligned}
$$

- $\quad$ flight time 


$$
\begin{aligned}
\text { Time } & =(\text { Battery capacity } / \text { Current draw }) \times 60 \\
& =(2.2 / 12.45) \times 60 \\
& =10.60 \text { minutes }
\end{aligned}
$$

\section{Development of QuadDrone for height measurement}

\subsection{CAD modelling}

Designing is the most important stage of any product development. The proposed QuadDrone is designed using SolidWorks as shown in Figure 3. Initially, the part drawings are made to the dimension specified and then the parts are assembled. The reason for choosing SolidWorks over AutoCAD is the fact that the ease with working and design complex model. The 2D modelling of drone is shown by Figure 4.

Figure 3 SolidWorks model - 3D printed drone frame (see online version for colours)

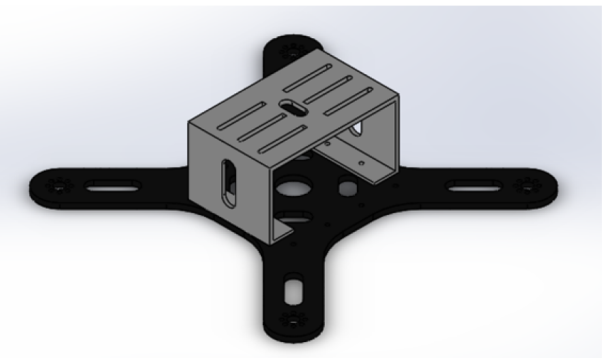

Figure 4 2D model (see online version for colours)

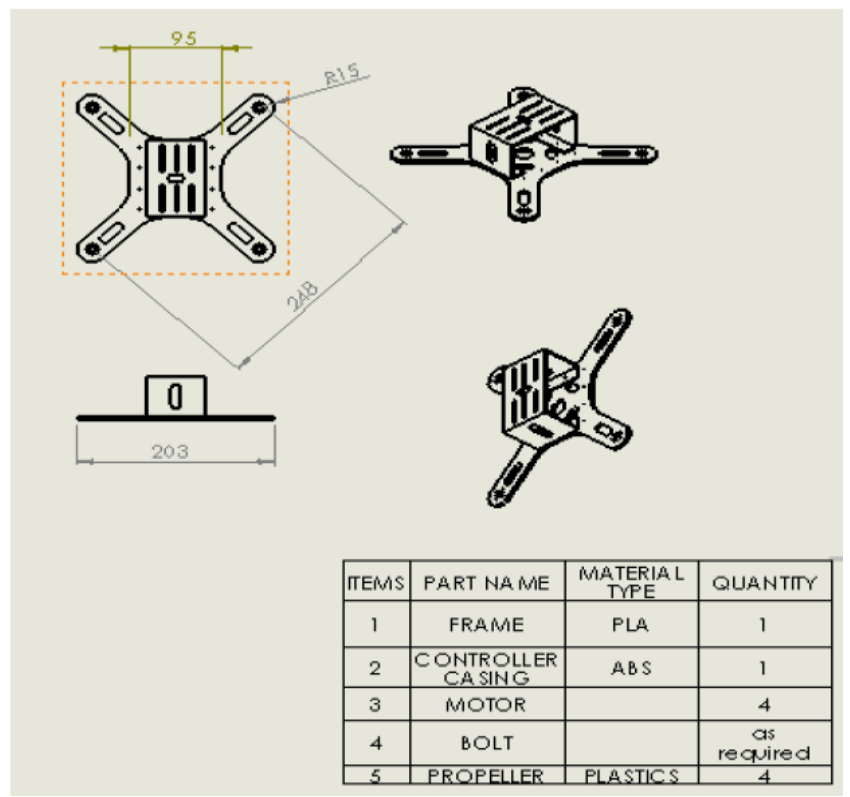




\subsection{Electrical interfacing}

The electrical parts of the drone consist of flight controller, receiver, BLDC motor, ESC, BMP180, Arduino Nano and Bluetooth module. The receiver is connected with KK2 flight controller in PWM signal generating pin. The controller controls the BLDC motor when user gives the input signal through transmitter and output produced in same controller to motor connection pin. The whole setup is powered with LiPo battery. The electrical circuit interfacing is shown in Figure 5.

Figure 5 Electrical interfacing (see online version for colours)

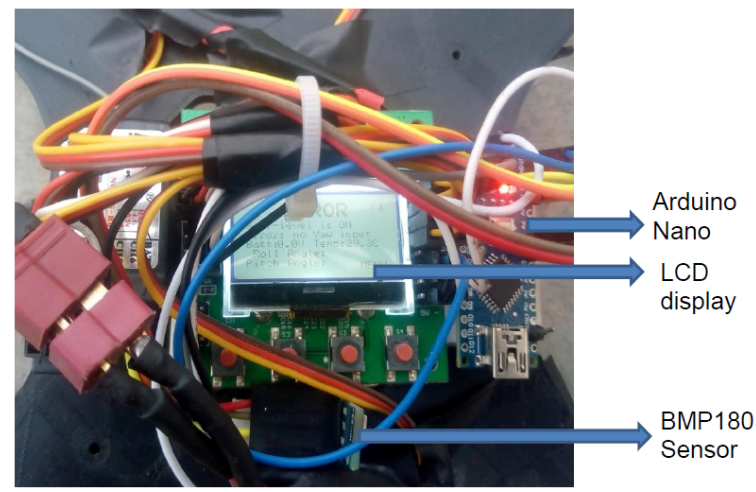

\subsection{Mechanical structure}

The 3D printed drone (Hell et al., 2018) frame is developed by considering the motor and propeller diameter ( 5 inch). Motor mount is used to fix the motor with frame. The frame size is about $760 \mathrm{~mm}$ which is obtained from the distance between two motors diagonally. Under the frame, the landing box is placed and it is also used for carrying battery sources. The propeller is mounted on the motor in alternate direction to eliminate torque produced by motor. The complete mechanical structure is shown by Figure 6 .

Figure 6 Mechanical structure (see online version for colours)

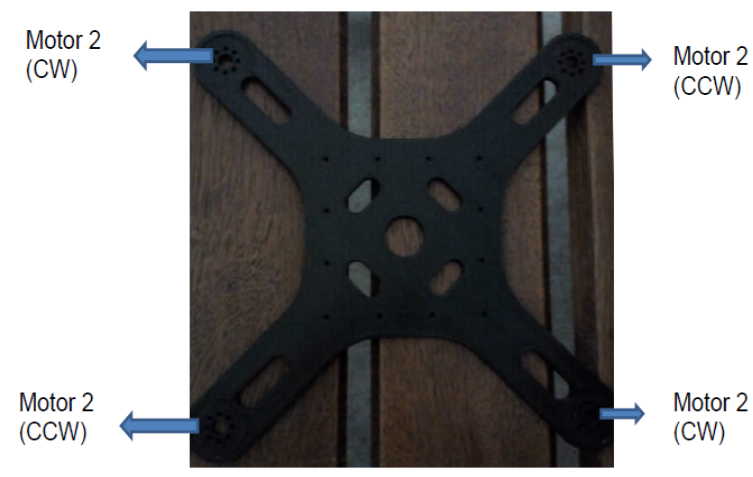




\subsection{Fabricated model}

The fabricated 3D printed QuadDrone consists of both mechanical and electrical components. The mechanical section consists of 3D printed parts are used as drone frame to fix the BLDC motors, LiPo battery, flight controller, and all other electronics components. After fabrication, accelerometer and gyroscope are calibrated and PID controller of KK2 flight controller is tuned for stability. The fabrication model is shown by Figure 7.

Figure 7 Fabricated 3D printed QuadDrone (see online version for colours)

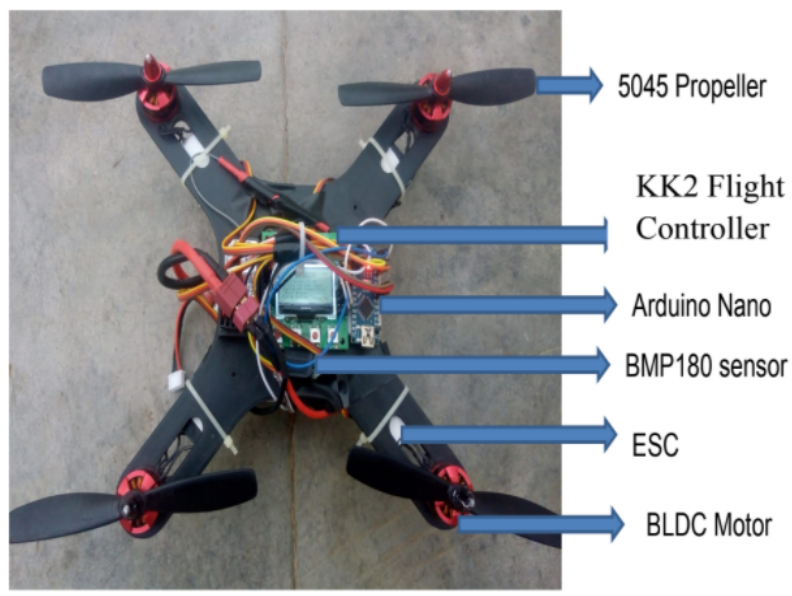

Figure 8 Functional diagram of 3D printed quadrone

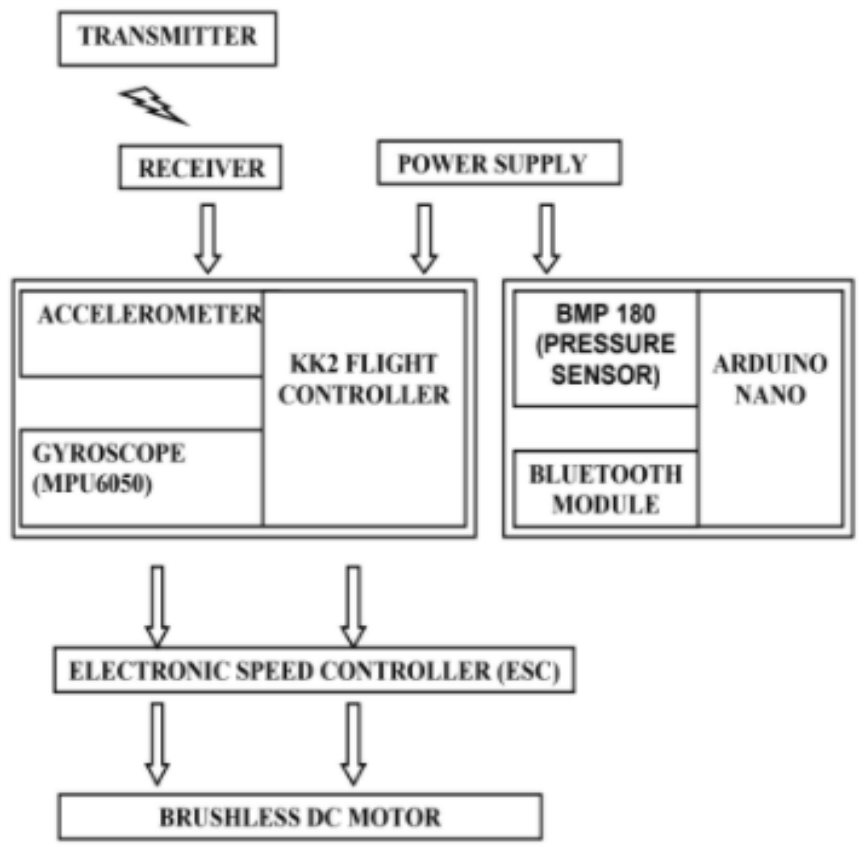


Figure 9 Photographic view of flying 3D printed QuadDrone (see online version for colours)

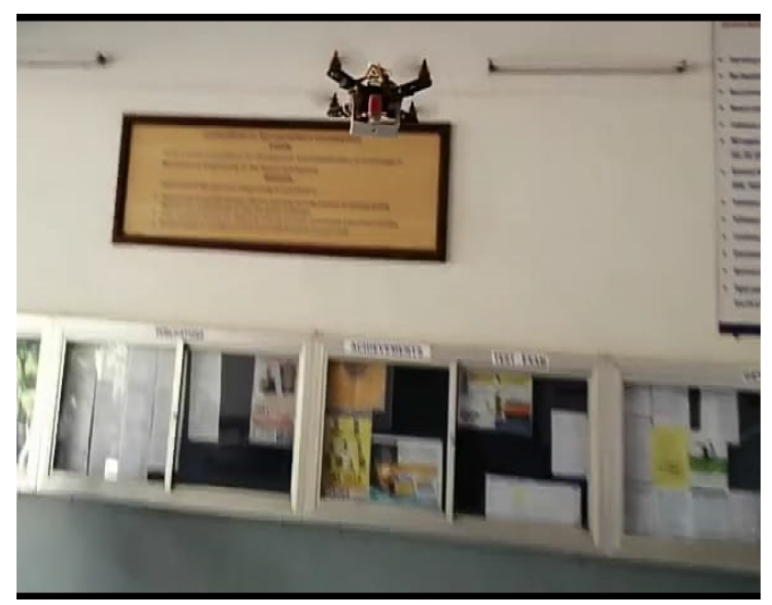

\subsection{Working principle}

The UAV is equipped with four brushless DC motors controlled by an ESC with the help of the KK2 flight controller. The inbuilt inertia measurement unit sensor (MPU6050) is used to control the orientation and stability of the drone as shown in Figure 8. When powered by a drone battery, the flight controller is enabled to calibrate the ESC and IMU. After calibration, the drone is ready to fly in the sky as shown in Figure 9. With the help of a transmitter, the drone is controlled by increasing the throttle stick to $50 \%$ of the speed of the motor. The Arduino Nano controller is connected to Bluetooth modules and the BMP180, which are used to measure height by measuring the pressure in their surroundings. As shown in Figure 10, the mobile phone is used as a display device to show the output measured from the BMP180 through the Bluetooth module.

Figure 10 Altitude display in mobile phone (see online version for colours)

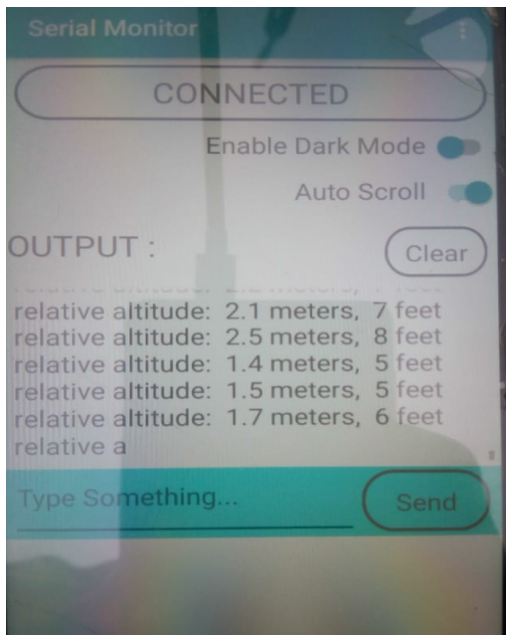




\section{Conclusions}

The main motivation of this project is to develop a 3D printed QuadDrone that can be used in height measurement. To control the drone, a $2.48 \mathrm{GHz}$ radio frequency transmitter, receiver, Arduino Nano microcontroller, ESC and brushless DC motor were used. Additionally, the BMP180 pressure sensor is equipped to measure height. SolidWorks has been used to develop drone frame structure design and analysis. The proportional, integral controller action reflects the improved performance of the roll, pitch and yaw of developed drone. Live altitude measurement was also demonstrated which shows the successful operation of drone tracking and data transmission from drone. These data are used for operator to determine the altitude in ground station.

\section{References}

Anthony, D., Elbaum, S., Lorenz, A. and Detweiler, C. (2014) 'On crop height estimation with UAVs', IEEE/RSJ International Conference on Intelligent Robots and Systems.

Bilewski, M. and Putynkowski, G. (2016) 'Application of ultrasonic distance sensors for measuring height as a tool in unmanned aerial vehicles with a stabilized position in the vertical plane', Scientific Journals Zeszyty Naukowe of the Maritime University, Vol. 46, No. 118, pp.17-21, Szczecin Akademii Morskiej w Szczecini.

Ganesh, Y., Raju, R. and Hegde, R. (2015) 'Surveillance drone for landmine detection', International Conference on Advanced Computing and Communications.

Hell, M., Bolam, R., Vagapov, Y. and Anuchin, A. (2018) 'Design of a portable drone for educational purposes', in Proc. 25th Int. Workshop on Electric Drives: Optimization in Control of Electric Drives (IWED), Moscow, Russia, 31 January-2 February, pp.1-5, DOI: 10.1109/IWED.2018.8321377.

Kale, S.D., Khandagale, S.V., Gaikwad, S.S. Narve, S.S. and Gangal, P.V. (2015) 'Agriculture drone for spraying fertilizer and pesticides', International Journal of Advanced Research in Computer Science and Software Engineering, Vol. 2, No. 6, pp.34-36. 